\title{
Royal College of Physicians of Edinburgh consensus conference on medical management of stroke, 26 and 27 May 1998
}

CONSENSUS STATEMENT

(1) The high incidence and serious consequences of stroke make it one of the most important challenges faced by contemporary medicine. In the UK stroke is one of the three main causes of death and a major cause of long term disability. As a result it consumes more NHS resources than any other condition.

(2) At a conference convened by the Royal College of Physicians of Edinburgh, a consensus panel considered four specific issues relating to the medical management of cerebrovascular disease in the UK. This statement is based on published research, augmented by presentations given at the meeting and expert opinion.

(3) The treatment recommendations in this consensus statement apply only to ischaemic events. Cerebral haemorrhage will need to be excluded before treatment is initiated. Whenever feasible this should be done as soon as possible with a CT scan.

What is the role of antiplatelet therapy in stroke?

(4) The beneficial role of antiplatelet agents in patients with stroke has been clearly established.

\section{(5) Acute stroke}

In acute stroke aspirin is the only proven antiplatelet agent. It should be commenced as soon as the diagnosis of cerebral infarction has been made, using a starting dose of 150 $300 \mathrm{mg}$ a day and continuing until decisions have been made about secondary prevention.

(6) Secondary prevention

In patients with prior ischaemic stroke or transient ischaemic attack (TIA) treatment should be with 75-300 mg aspirin, continued long term.

(7) There is evidence that clopidogrel and the combination of aspirin and modified release dipyridamole are safe and effective alternatives to aspirin alone. There is also some evidence that they may be more effective than aspirin alone, but this evidence does not yet establish sufficient additional benefit to justify their adoption as first line treatment, particularly in view of their cost. Further randomised controlled trials (RCTs) of both their effectiveness and cost effectiveness are required.

(8) Patients genuinely intolerant of aspirin should be given modified release dipyridamole, or clopidogrel when licensed.

WHAT IS THE ROLE OF ANTICOAGULANT THERAPY IN STROKE?

\section{(9) Acute stroke}

There is no evidence to support the use of anticoagulants for the treatment of acute stroke, even in patients in atrial fibrillation Anticoagulation is not associated with an overall reduction in death or disability as a decrease in recurrent ischaemic stroke is offset by an increase in haemorrhagic stroke. There is also a significant excess of extracranial bleeds. Although there is good evidence that heparin (including low molecular weight heparin) does prevent deep vein thrombosis (DVT), the risk of fatal pulmonary embolism is lower than the risk of intracranial haemorrhage in these patients. Physical methods of preventing DVT in stroke patients should therefore be evaluated. Symptomatic venous thromboembolism complicating stroke should be managed according to established guidelines.

\section{(10) Secondary prevention}

Patients who have had a TIA or an ischaemic stroke and are in atrial fibrillation should be considered for long term treatment with warfarin (suggested target INR 2.5) as this greatly reduces the long term risk of embolic stroke. If there is a contraindication to warfarin, aspirin should be used. The optimal timing for the initiation of anticoagulation after the acute event is unresolved. In order to minimise the risks of cerebral haemorrhage the initial treatment should be with aspirin until, for example, the majority of the stroke deficit has resolved or, in the case of more severe strokes, more than 2 weeks have elapsed. At present there is no evidence to support the use of warfarin in most patients in sinus rhythm, although there are exceptions such as patients with mechanical heart valves. Warfarin and aspirin should only be given together in exceptional circumstances.

WHAT IS THE ROLE OF THROMBOLYTIC THERAPY IN STROKE?

(11) Intravenous thrombolytic therapy is potentially an effective treatment of acute stroke, offering the possibility of early reperfusion of ischaemic cerebral tissue and limitation of infarct size. However, all thrombolytic drugs need to be given early after the onset of symptoms (probably within 6 hours) and involve a risk of cerebral haemorrhage. There may also be an adverse interaction between streptokinase and aspirin

(12) Data from 12 RCTs, involving three drugs (streptokinase, urokinase, and recombinant tissue plasminogen activator) and fewer than 4000 subjects, are currently available. The results suggest an increase in the proportion of patients making a good recovery by 6 months but a substantial increase in cerebral haemorrhage within the first 2 weeks. There are insufficient data available at present to determine the optimal drug and dosage required, although the situation may be clearer within 12 months.
(13) Although the potential benefits of thrombolytic therapy are substantial, so too are the risks and the service implications. For this reason we consider that these drugswhether licensed or unlicensed-should not normally be prescribed on present evidence except in the context of an RCT.

(14) There is an urgent need for a large, multicentre RCT of thrombolytic therapy. This will require extensive organisational changes if adequate numbers of patients are to meet the 6 hour deadline.

WHAT IS THE ROLE OF CAROTID SURGERY IN STROKE?

(15) Carotid endarterectomy has a role in preventing stroke in patients with recent (within 6 months) carotid territory symptoms in association with severe stenosis of the ipsilateral carotid artery, and who are fit for surgery. Surgery should be targeted at patients at highest risk of further stroke (such as those with frequent TIAs, cerebral rather than ocular symptoms, ulcerated rather than smooth stenosis) and performed as soon as possible after the initial event. Delays in the process of identifying and investigating patients who might benefit from surgery should be minimised by rapid access to Duplex ultrasound, CT, and angiography.

(16) Surgery should be restricted to specialist centres to minimise perioperative stroke and deaths; regular audit is essential.

(17) Carotid angioplasty with or without stenting may offer an alternative to surgery in selected patients and probably carries similar operative risks. Further RCTs are essential before this technique comes into routine use, to establish its safety, effectiveness, and cost effectiveness.

CONCLUDING REMARKS

(18) We emphasise the importance, and urge the further development, of well organised and coordinated stroke services. There is good evidence that these lead to improved patient outcomes. The elimination of delays in presentation, referral, and investigation, and in the initiation of treatment and rehabilitation, are all likely to lead to further improvements.

(19) Although $40 \%$ of stroke patients are over 80 years of age, few clinical trials have included many patients of this age. We can only suggest extrapolation of trial results to the elderly, recognising that both the risks and benefits may differ. We also recommend that as many elderly patients as possible should be included in future trials.

(20) We have considered the four questions we were asked to address. It is also important not to neglect all the other measures which contribute to optimal management, including the detection and effective treatment of 
hypertension, diabetes and hyperlipidaemia, and smoking cessation.

(21) Stroke patients are particularly vulnerable, and clinicians should bear in mind the legal and ethical reasons for discussing risks, benefits, and alternatives with them, and where possible obtaining valid consent. This is particularly important in the context of clinical trials.

(22) Patients with cerebrovascular disease are cared for by a variety of healthcare workers, in both primary and secondary care settings. We urge these clinicians in each locality to meet to consider these recommendations and incorporate them into their own guidelines and practice.
CONSENSUS PANEL

Dr John M Bamford, neurologist and cerebrovascular physician, St James University Hospital, Leeds.

Professor Senga Bond, professor of nursing research, Centre for health Services Research, University of Nottingham.

Mr David H Clark, chief executive, Chest, Heart, and Stroke, Scotland.

Dr Andrew T Elder (vice chairman), consultant physician in geriatric medicine, Western General Hospital, Edinburgh.

Professor Michael Greaves, professor of haematology, University of Aberdeen.

Dr Martin C G Hadshar, general practitioner, Coldstream.
Mr Paul Haines, science editor, hospital doctor, Reed Healthcare Publishing, Sutton, Surrey.

Professor Robert E Kendall (chairman), President, The Royal College of Psychiatrists, 17 Belgrave Square, London.

Professor Sheila A M McLean, International Bar Association Professor of Law and Ethics in Medicine, University of Glasgow.

Professor Ian T Russell, Founding Professor of Health Sciences and Clinical Evaluation, University of York.

Mr George $\mathrm{H}$ Welch, consultant vascular surgeon, Glasgow South Vascular Surgery, Southern General Hospital NHS Trust. 